\section{$\underset{\substack{\text { hommes } \\ \text { \& migrations }}}{ }$}

\section{Hommes \& migrations}

Revue française de référence sur les dynamiques

migratoires

\section{$1320 \mid 2018$}

Au prisme de la consommation

\title{
La « prairie thaï » dans le Preußenpark à Berlin
}

Une communauté de pique-nique en marge du règlement intérieur des parcs allemands

\section{Noa K. Ha}

\section{(2) OpenEdition}

\section{Journals}

Édition électronique

URL : http://journals.openedition.org/hommesmigrations/4040

DOI : 10.4000/hommesmigrations.4040

ISSN : 2262-3353

Éditeur

Musée national de l'histoire de l'immigration

Édition imprimée

Date de publication : 1 janvier 2018

Pagination : 13-19

ISBN : 978-2-919040-40-7

ISSN : $1142-852 X$

Référence électronique

Noa K. Ha, «La « prairie thaï » dans le Preußenpark à Berlin », Hommes \& migrations [En ligne], 1320

2018, mis en ligne le 01 janvier 2021, consulté le 07 janvier 2021. URL : http://

journals.openedition.org/hommesmigrations/4040; DOI : https://doi.org/10.4000/

hommesmigrations.4040 


\title{
LA «PRAIRIE THAÏ » DANS LE PREUSSENPARK À BERLIN UNE COMMUNAUTÉ DE PIQUE-NIQUE EN MARGE
DU RĖGLEMENT INTÉRIEUR DES PARCS ALLEMANDS'
}

Par NOA K. HA, chercheuse posdoctorante, Institut des sciences de l'art et de l'urbanisme historique, université technique de Berlin (TU).

\begin{abstract}
Le Preußenpark, à l'ouest de Berlin, est devenu un rendez-vous incontournable des couples germano-thaïlandais où de nombreuses « épouses thaï » partagent en plein air leur savoirfaire en matière de cuisine asiatique. Le parc est devenu tout autant un espace de détente pour les couples mixtes qu'un lieu de rendez-vous exotisé où se croisent, non sans difficultés, les cultures thaï et allemandes. Derrière les plats, à même le sol, se jouent des enjeux de pouvoir au sein même des couples et à l'égard de la société dominante. Dans les deux cas, il s'agit pour les femmes d'origine thaillandaise de cultiver ce territoire de leur autonomie contre les discriminations qui les visent.
\end{abstract}

À Berlin, le Preußenpark ${ }^{2}$ est une adresse que rien ne distingue et que l'on se repasse pourtant comme un bon tuyau de lieu insolite. Sa particularité tient au fait que l'une de ses grandes et vastes pelouses (ou prairies), ceinte d'arbres majestueux, est fréquentée par de nombreuses personnes d'origine asiatique, avant tout par des femmes thaïlandaises. L'été, jusqu'à 500 personnes s'y retrouvent le weekend (voir photo 1), ouvrent des parasols, sortent réchauds et plaques de cuisson, installent du matériel de camping, coupent, grillent, mitonnent, cuisent à la vapeur légumes, viandes et poissons, jouent aux cartes, vendent des produits alimentaires et passent tranquillement leur week-end sur une couverture étalée à même le sol. Les réchauds et plaques de cuisson sont montés directement sur la pelouse, et il est recommandé de s'accroupir pour savoir ce qui se mijote dans les marmites et combien cela coûte. Certains plats sont préparés à l'avance, d'autres sont cuisinés sur place. Som Tam ${ }^{3}$, currys, sushis, rouleaux de printemps, variations de poissons, viandes, légumes et riz sont au menu, volontiers accompagnés d'une bière ou d'une caïpirinha mixée sur commande. Les arbres verts à l'arrière-plan et les parasols bigarrés composent un paysage unique : soleil, senteurs et bruits font vagabonder les pensées au loin - souvenirs du pays natal pour les uns, désir de vacances pour les autres.

Les observations qui suivent sont produites depuis ma position de femme asiatique allemande qui visite occasionnellement le parc thaï avec sa famille et $\mathrm{y}$ rencontre des amis autour d'un repas 
savoureux. Mon propre milieu communautaire est très différent de ceux qui se rencontrent et font du commerce dans le parc. Néanmoins, nous partageons une même appartenance raciale et genrée qui est perçue dans la société allemande comme la figure de l'Autre, celle, exotique, soumise et passive, des femmes asiatiques ${ }^{4}$. Je ne fais pas partie de la communauté thaï, mais je suis membre d'une organisation de jeunes germano-asiatiques de la deuxième et de la troisième génération qui lutte contre les stéréotypes raciaux présents dans les médias populaires, contre les formes d'exclusion liées à la citoyenneté ou à la migration de travail ${ }^{5}$. La migration d'Asie vers l'Allemagne a une longue histoire. Structurée selon les différents points géographiques de départ en Asie, elle s'incarne dans différents contextes transnationaux comme, par exemple, entre l'Allemagne et la Chine ou l'Allemagne et l'Inde, l'Allemagne et la Corée du Sud, l'Allemagne et le Vietnam, et bien d'autres encore ${ }^{6}$. Me situant dans la société allemande, mes recherches visent à rendre compte des processus d'exclusion visibles par

4. T. Minh-ha Trinh, Woman, Native, Other: Writing Postcoloniality and Feminism, Bloomington, Indiana University Press, 1989. 5. Le nom de l'organisation est « Korientation ». Elle a été fondée en 2008 par des jeunes germano-coréens de la deuxième génération. Leur objectif était de créer une organisation capable de les représenter véritablement et qui lutterait contre des discours stéréotypés et arriérés présents au sein de la société allemande sur les migrants asiatiques. 6. Pipo Bui, Envisioning Vietnamese Migrants in Germany: Ethnic Stigma, Immigrant Origin Narratives and Partial Masking, Münster, Lit Verlag, 2003 ; Dagmar Yu-Dembski, Chinesen in Berlin, Berlin, Be. Bra Verlag, 2007 ; Jae-Hyeon Choe, Hansjürgen Daheim, Rückkehr-und Bleibeperspektiven Koreanischer Arbeitsmigranten in der Bundesrepublik Deutschland, Francfort, Peter Lang, 1987 ; Urmila Goel, Jose Punnamparambil, Nisa Punnamparambil-Wolf (dir.), InderKinder: über das Aufwachsen und Leben in Deutschland, Heidelberg, Draupadi, 2012. 
la « race », l'ethnicité, la sexualité, le genre, la classe sociale et d'autres catégories normatives liées au corps humain. C'est pourquoi mon positionnement académique est fermement inscrit dans le champ des études féministes postcoloniales et décoloniales ${ }^{7}$, qui contestent la notion d'« objectivité » en recontextualisant les connaissances situées ${ }^{8}$.

\section{Pique-niquer et fabriquer un « chez soi »}

Les premiers « rendez-vous thaï » au Preußenpark remontent au milieu des années 1990. L'ethnologue Karl Braun a relaté le mythe fondateur des quatre familles germano-thaï qui s'y seraient régulièrement retrouvées pour y pique-niquer ${ }^{9}$. Elles auraient ensuite été rejointes par des femmes et des hommes d'autres communautés asiatiques de la ville, mais également par de nombreux Berlinois et touristes. Ce sont des femmes, avant tout, qui agencent le parc selon leurs besoins, y cuisent et vendent leurs préparations culinaires. Des femmes viennent de Thaïlande, mais aussi des Philippines ou du Vietnam. Beaucoup d'entre elles se sont rendues en Allemagne pour se marier et ont épousé des Allemands blancs. Comme on peut le voir sur la photo 2, ces femmes sont assises au premier rang, des hommes d'origine allemande ou asiatique les secondent à côté ou derrière elles. Également assis, ils taillent des légumes; parfois, ils se lèvent pour porter des boissons, déballent et emballent des boîtes dans lesquelles ils ont transporté les ingrédients ou les plats tout prêts, ou expliquent aux chalands les plats proposés à la vente. D’autres hommes blancs sont assis en groupe à lécart, une bouteille de bière à la main, et parlent peut-être, de leur dernier voyage en Thaillande. Il se dégage à première vue de cet ensemble une impression idyllique d'entreprise familiale interculturelle. Cependant, les arrangements matrimoniaux germano-thailandais sont sous-tendus par des rapports fragiles. Nivedita Prasad, ancienne chercheuse au sein de l'association Ban Ying-Beratungs-und Koordinationsstelle gegen Menschenhandel (Association de conseil et de coordination contre la traite humaine) et professeure à l'établissement d'enseignement supérieur AliceSalomon-Hochschule, évoque une facette moins visible : beaucoup de femmes cherchent le dimanche à sortir de la solitude de leurs appartements dans lesquels leurs époux allemands tentent de décider des menus à consommer, de la langue parlée aux enfants en fonction de l'occasion ou encore des pratiques culturelles appropriées, sans même parler des violences conjugales présentes dans certaines de ces relations. Nivedita Prasad fait ainsi valoir combien il est étonnant que ces femmes aient réussi à créer leur propre espace féminin dans le Preußenpark.

Le week-end, elles s'aménagent un espace de liberté dans leur quotidien interculturel avec leurs maris allemands, espace qui, dans le parc, devient public et dévoile indirectement une "normalité » dans les rapports de genre germano-asiatiques. Si nous considérons les arrangements matrimoniaux germano-asiatiques comme une forme de migration du travail mondialisée, alors se démultiplient et se chevauchent ici les conditions globalisées du travail reproductif sexué et du travail relationnel racialisé. Dans cet espace, les hommes allemands blancs se font les complices de la reproduction de rapports de pouvoir qui exploitent la pauvreté féminine de l'hémisphère Sud. Les femmes, quant à elles, développent des stratégies au sein de ces interdépendances, pour réaliser leurs propres objectifs 
existentiels et se constituer une sécurité sociale au sein de leurs réseaux. Dans la perspective de la société dominante allemande, les femmes asiatiques sont soit exotisées comme des objets de désir, soit conçues comme des victimes de leur histoire. Elles sont rarement envisagées comme des actrices de leur propre destinée. Pourtant, c'est bien à cette expérience que sont confrontés les Allemands blancs quand leurs préjugés à l'égard des femmes asiatiques - "gentilles, dociles et casanières » - ne sont pas corroborés. De nombreuses femmes, en effet, prennent les choses en main au sein du foyer et rappellent leurs époux à leurs devoirs d'aider financièrement leurs familles au pays. C'est aussi le constat auquel est parvenue Andrea Lauser dans ses recherches ethnographiques : elle a étudié les relations matrilinéaires et découvert que ces femmes venues pour se marier en Allemagne sont des migrantes faisant preuve de beaucoup de dynamisme et qui ont construit activement leur vie. Bien que la précarité de leur situation initiale limite leurs possibilités d'agir, elles mettent en place des structures solidaires et se forgent une position. C'est une des raisons pour laquelle Andrea Lauser reprend une citation des femmes interrogées comme titre de son ouvrage: 
"Un homme bon demande beaucoup de travail ${ }^{10}$ ", et renvoie à leur construction active de leurs conditions de vie. En ce sens, la longue histoire de la « prairie thaï » dans le Preußenpark de Wilmersdorf à Berlin raconte une histoire de femmes asiatiques qui sont des productrices actives de l'espace.

\section{Le règlement intérieur du Preußenpark}

Le « règlement du parc » est affiché dans une petite vitrine à l'entrée, à côté d'un plan en trois langues : allemand, anglais et thaïlandais. Le premier règlement plurilingue a été rédigé dès 1997 et stipule : "Le Preußenpark est un espace vert public destiné à la détente des riverains et des visiteurs. L'utilisation du parc doit se faire dans le respect. Les plantations et équipements ne doivent subir aucun dommage, salissure ou quelconque entrave et les autres visiteurs du parc comme les riverains ne doivent pas être mis en danger. Merci de noter qu'il est autorisé de faire des barbecues, de cuire ou de préparer des repas dans les aires prévues à cet effet le long de la Württembergische Straße jusqu'à 22 heures uniquement. Il est admis de s'allonger et de pique-niquer sur toutes les aires engazonnées.

Il est interdit :

1. De faire des barbecues, de cuire, mijoter et d'allumer ou d'entretenir un feu hors de la zone de barbecue.

2. De déployer du mobilier de jardin (par ex. tables, chaises, tentes et parasols).

3. De disposer plus de glacières que ne l'exigent les quantités usuelles de sa propre consommation.

4. De vendre des produits et de proposer des prestations de service.

5. De venir avec des chiens ou d'autres animaux domestiques (merci de ramasser immédiatement les crottes de chien).

6. De faire du vélo.

7. De faire du bruit, par ex., au moyen d'instruments de musique ou d'appareil de diffusion sonore, dès que d'autres visiteurs en sont gênés de manière excessive.
8. De pénétrer sur les pelouses après 22 heures.

Administration de Charlottenburg-Wilmersdorf, département des ouvrages publics, service des espaces verts, tél. : 9029-14488. »

Le libellé de ce règlement, qui se distingue en certains points d'autres réglementations de parcs et jardins, montre très clairement que les pratiques de pique-nique décrites plus haut sont considérées comme inopportunes et qu'il s'agit de protéger les riverains - des citoyens blancs pour la plupart. Dans ce règlement se dessine une ligne de clivage qui ressurgit quasiment tous les étés. Si les tensions entre autorités locales et " communauté de pique-nique » ont atteint des sommets entre 1997 et 2000 , et que la police a procédé à une razzia contre les pratiques d'utilisation du parc à l'été 2000, des solutions ont par la suite été trouvées concernant l'hygiène, l'enlèvement des déchets, les nuisances sonores et l'utilisation des toilettes publiques. Le règlement du
Dans cet espace, les hommes allemands blancs se font les complices de la reproduction de rapports de pouvoir qui exploitent la pauvreté féminine de l'hémisphère Sud. parc n'a pas été uniquement affiché sur place, il a également circulé au sein de la communauté germano-thailandaise. Sa publication a été relayée par le magazine Farang - Das Südostasienmagazin aus Berlin, un mensuel qui diffuse depuis 1997 les actualités de la communauté et a été fondé par des conjoints allemands se retrouvant régulièrement au parc.

\section{Une présence remise en question}

Les conflits avec les services municipaux chargés de l'ordre ou avec la police sont un thème récurrent et d'une grande importance au sein de la communauté. Le récit des contrôles est produit par des Allemands qui se sentent personnellement agressés par ce clivage entre le maintien de l'ordre du parc et les activités indésirables de « leurs femmes thaï ». Sous le titre "La section des vétérans s'essaie à la rébellion - les services d'ordre interviennent », ils 
prennent position comme suit dans leur magazine : "Mais jeter d'emblée le bébé avec l'eau du bain, c'est-àdire interdire par principe de s'asseoir sur des meubles de camping légers amenés par ses propres soins - même de toute petite taille - là, on doit s'insurger. Cela sent un peu trop la chicane et les abus des pouvoirs publics! Il y a plus de 16 (en toutes lettres : seize) ans que des Thai et des Allemands ont commencé à se retrouver au Preußenpark et ont découvert ce lieu de détente au cour de la cité. La presse s'en est fait l'écho dans le monde entier comme sur Internet et la destination " parc thaï " est même proposée dans certains voyages organisés. Depuis le début, les Thaïs et les Allemands ont apporté leurs tabourets ou chaises basses, glacières et tables de camping et tout ce dont on a besoin pour pique-niquer. Jamais cela n'a soulevé de question jusqu'au printemps 2006 ! Puis, tout d'un coup, il n'est plus admis d'installer du "mobilier de jardin"?! On ne peut s'empêcher de soupçonner que les individus qui,

L'importance du parc excède de loin les frontières du voisinage et renferme un vaste paysage de sens enchâssé entre tourisme international, migration par le mariage et gastronomie. au sein des services municipaux, ont de tout temps été contre la direction prise par le "parc thai", ont voulu ici, pour ainsi dire, faire table rase d'un seul coup! Restaurer sciemment le statu quo ante sous couvert de chasser les vendeurs à la sauvette, avec pour devise : le Preußenpark aux seuls riverains ${ }^{11}$ ! » On relève ici un attachement à sa "propre " communauté et aux retrouvailles régulières dans le « parc thaï ». L'importance du parc excède de loin les frontières du voisinage et renferme un vaste paysage de sens enchâssé entre tourisme international, migration par le mariage et gastronomie. Le " parc thaï » est aujourd'hui fort d'une histoire de près de deux décennies, mais son avenir est flou. La presse berlinoise colporte presque chaque année la rumeur d'une intervention des autorités locales et de l'interdiction de ce marché. En 2001, par exemple, le tabloïd B.Z. titrait : «Preußenpark : le parc thaï de Berlin doit disparaître ». D'un autre côté, la " prairie thaï » a acquis une notoriété qui se lit dans les reportages médiatiques internationaux, les encarts publicitaires et les notations dans les guides gastronomiques, faisant de ce marché une institution de la ville.

\section{Un espace public de partage des cultures}

Le « parc thaï » donne le sentiment d'être un joyau qui séduit par une authenticité de carte postale. Un foodmarket apparemment "authentique », tel qu'on en trouve immanquablement dans les villes d'Asie, qui convie au pique-nique. Il permet aux visiteurs de goûter pour une somme modique à des plats thailandais ou vietnamiens frais et issus de recettes familiales, et de faire, en plein cœur de Berlin, une brève excursion en "Extrême-Orient ". Toutefois, la perspective blanche de "l'authentique » et de "l'exotique», qui donne au parc des airs de "petit Bangkok" ou offre de faire une découverte fascinante de la "cuisine extrêmeorientale », occulte la position précaire des femmes de ce continent.

Si la prairie thaï du Preußenpark existe, c'est parce que beaucoup de personnes ont créé ensemble un espace qui, à leurs yeux, renvoie aux saveurs et aux langues de leurs pays d'origine. Cet espace existe parce que des femmes partagent et transmettent leurs savoirs et acquis. Ces pratiques donnent lieu à des expériences communes : cuisiner de concert, jouer et parler ensemble ou encore passer le weekend dans une convivialité détendue. Les piqueniques communs dans l'espace public défient non seulement l'administration municipale, mais interrogent aussi la pertinence de ces espaces pour la constitution de la communauté. L'existence du " parc thaï » atteste que ces femmes exploitent une possibilité informelle et délibérément choisie d'échanges et d'interconnexions. Il s'agit là d'un aspect extrêmement important pour peu que l'on prenne en compte la position défavorisée dans laquelle elles se trouvent. L'administration n'y prête manifestement pas grand intérêt, parce qu'elle a tendance à considérer la signification 
interculturelle des espaces publics comme secondaire et à accorder la priorité aux intérêts des riverains comme aux exigences d'hygiène d'un parc allemand.

Bien que la création de "jardins interculturels » atteste d'une supposée diversification des catégories d'usagers du paysage de relaxation berlinois, les interdictions de barbecues, dans d'autres parcs de Berlin comme celui du Tiergarten ${ }^{12}$, par exemple, limitent précisément les possibilités de détente de familles d'origine turque ou arabe à faibles ressources et confinées dans de petits appartements. Par ailleurs, les communautés immigrées ont un accès restreint aux associations de jardins ouvriers [très répandus à Berlin, NDT], parce que ces dernières ne brillent pas particulièrement par leurs compétences interculturelles. De même, leurs statuts compliqués ont un effet dissuasif. Enfin, les migrants, comme dans l'association Kleingartenverein Norderstedt, ne sont autorisés à y adhérer qu'en nombre réduit ${ }^{13}$. La difficulté rencontrée par les communautés de migrants à instaurer leurs propres espaces et formes de détente dans l'espace public et à élargir l'ouverture interculturelle des espaces verts allemands révèle des déficits urbains, peu pris en compte à ce jour.

Dans ce contexte, on est finalement surpris par l'existence prolongée de la communauté de piquenique du " parc thaï » et du Tiergarten, entreprise communautaire constamment menacée d'être criminalisée mais qui raconte dans le même temps une nouvelle histoire du Preußenpark. Sur le plan symbolique, on peut lire cette histoire comme une narration d'une époque (singulière) en Allemagne, dans laquelle certains symboles culturels dominants sont réécrits dans les pratiques du quotidien de communautés marginalisées. Si le règlement allemand des parcs ne s'est pas encore adapté au quotidien interculturel dans le pays d'immigration, ce quotidien s'y est toutefois frayé un chemin depuis longtemps et montre, au travers de facultés d'adaptation tout autant que de résistance, des voies pour créer ses propres espaces germano-asiatiques. 